\title{
Critical applications in vehicular ad hoc/sensor networks
}

\author{
Neeraj Kumar • Al-Sakib Khan Pathan • \\ Elias P. Duarte Jr. · Riaz Ahmed Shaikh
}

Published online: 14 November 2014

(C) Springer Science+Business Media New York 2014

Vehicular ad hoc networks (VANETs) have emerged as a promising technology with several applications that may have a deep impact on our society. In the past few years, we have witnessed a tremendous growth in this field, with several new techniques and standards having been proposed with the objective of making it easier and safer to drive and transport passengers. Diverse applications are already available that provide information on traffic conditions, the density of vehicles, duration of traffic lights, among many other variables that allow smarter driving decisions to be taken. Moreover, drivers and passengers can download and upload this information as they travel, with the aid of the Road Side Units (RSUs), some of which are already deployed. RSUs are usually connected to the Internet, and are thus widely available to users and services.

Among various kinds of advancements, today's sophisticated vehicles could be equipped with sensor nodes which could monitor and collect the data in sparse and dense regions. These data could be processed for the benefit of com-

N. Kumar $(\bowtie)$

Department of Computer Science and Engineering,

Thapar University, Patiala, Punjab, India

e-mail: neeraj.kumar@thapar.edu; nehra04@yahoo.co.in

\section{A.-S. K. Pathan $(\varangle)$}

Department of Computer Science, International Islamic University

Malaysia, Kuala Lumpur, Malaysia

e-mail: sakib.pathan@gmail.com

\section{E. P. Duarte Jr.}

Department of Informatics, Federal University of Paraná, Curitiba,

Brazil

e-mail: elias@inf.ufpr.br

\section{R. A. Shaikh}

Department of Computer Science, King Abdulaziz University, Jeddah, Saudi Arabia

e-mail: rashaikh@kau.edu.sa munities of people, for example, health monitoring and diagnostic systems, safety alarms, intelligent transport systems, and environment monitoring. The vehicles during movement, equipped with sensor nodes, can also collect the data from human body and that data can be processed in collaboration with the other vehicles for various medical purposes. In this case, VANETs can also be integrated with Body Sensor Networks.

However, as a new technology emerges, it throws new challenges to the research community. Although many solutions already exist focusing on different target areas, there still remains a need for more efficient solutions which can be used in wide variety of applications. Many industrial and academic institutions have already geared up to provide solutions for the benefit of the community. Moreover, there are many constraints that have to be resolved before providing any standard solution. Some examples of the constraints include; mobility of the nodes, connectivity in sparse and dense regions, failure of nodes, network traffic congestion and interference, etc.

The objective of this special issue was to compile papers on various topics in the fields related to the critical applications of Vehicular ad hoc/sensor networks. With successful dissemination of the call, we received in total 35 high quality submissions from around the globe. Each paper was carefully reviewed by a team of experts in the field. After a two-phase rigorous review process, only five papers among the 35 received submissions were selected for inclusion in this Special Issue. The main contributions of these accepted papers are summarized next.

Guerroumi et al. proposed a new Sink mobile and power management strategy for efficient data dissemination in Wireless Sensor Networks. The authors have described a new power threshold by introducing a sink mobility scheme to balance the network load between sensor nodes. In the 
proposed scheme, the sensor nodes are organized in clusters, and a cluster head is selected for each cluster. The cluster head collects and transmits sensing data using the proposed data dissemination process. In this phase, the sensor sink may move toward any cluster based on its sensing data frequency to minimize energy consumption of sensor nodes. The nearer the nodes are to the fixed sinks, the smaller the energy consumption is, due to the need of relaying large amounts of data. From the performance evaluation of the proposed scheme, the conclusion was reached that the proposed scheme outperforms other existing related approaches.

Malina et al. proposed a new efficient group signature for privacy preservation in VANETs. The authors first analyse the impact of several common attacks like Denial of Service or replay attacks on the efficiency of privacy-preserving security solutions in the vehicular networks. Then a privacy preserving scheme is specified, which is based on the group signatures. A formal security analysis is performed, and experimental validation is provided.

Misra et al. proposed a new strategy that is aware of selfish behavior for target tracking in mobile WiMAX-based Vehicular environments. The authors present a Selfishness Aware Target Tracking algorithm which employs the time difference of arrival measurement data when the target is in Line-ofsight (LOS) with more than three base stations (BSs). When no more than three LOS links between the target and the BSs are available, then the cluster-head which is currently serving the target, activates the three most promising mobile nodes for collecting location information about the target. The results obtained show that the scheme proposed by the authors is effective for target tracking in mobile WiMAXbased vehicular environments.

Ho et al. argue that the robustness of Vehicular networks can be enhanced using virtualization technology. The authors employ virtual routers and virtual links for designing failure resilient VANETs. The proposed scheme was able to retain more than $80 \%$ of the original capacity with only $50 \%$ nodes working in the network. The authors validate the proposed scheme both using simulation and analysis.

Finally, privacy-Aware access control for message exchange in VANETs is presented by Karumanchi et al. This work is the first one of this kind in which a comprehensive framework for access control in VANETs is proposed. The authors present illustrative scenario, details of vehicle attestation and registration procedures, and way of verifying vehicle's location. Alongside presenting their analysis and results, they also talk about policy issues. Overall, this is a good work that combines the advantages of previous schemes and suggests new directions toward privacy protection in VANET scenarios.

The entire process of preparing this special issue was long and we had to work non-stop. However, thanks must be given to the authors of all submitted papers for their interest in this special issue. We congratulate them on the various relevant research projects they are working on for which some results have already been achieved. Due to the strict review process, we could not accommodate all papers, even if of good quality. A comparative scale has been used to finalize the list of the papers. We are very grateful to all the reviewers, for their careful and timely works. Last but not the least, we are deeply grateful to Prof. Bezalel Gavish (Editor-in-Chief, Telecommunication Systems Journal) for providing us the opportunity to take role as guest editors of this special issue and for all the supports he has provided along the way. We hope the papers in this issue would be beneficial to the research community.

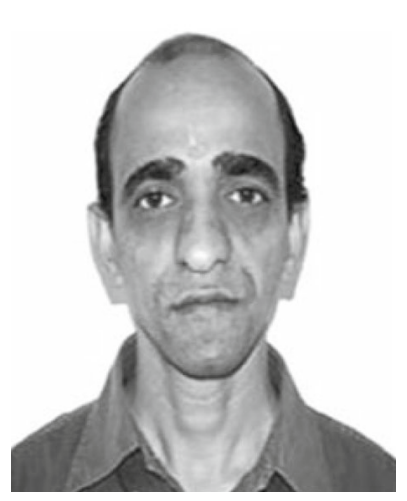

Neeraj Kumar is working as Associate Professor in Department of Computer Science and Engineering, Thapar University, Patiala, Punjab (India). He received his Ph.D. in CSE from Shri Mata Vaishno Devi University, Katra (India) and PDF from UK. He has more than 70 research publications in peer reviewed journals and conferences including IEEE, Elsevier, and Springer. His research is focused on mobile computing, parallel/distributed computing, multi-agent systems, service oriented computing, routing and security issues in wireless ad hoc, sensor and mesh networks. He is leading the Mobile Computing and Distributed System Research Group. Prior to joining Thapar University, Patiala, (Punjab), he has worked with HEC Jagadhri (Haryana) and MMEC Mullana, Ambala (Haryana), and SMVDU, Katra (J\&K). He has delivered invited talks and lectures in various IEEE international conferences in India and abroad. He has organized various special sessions in international conferences in his area of expertise in India and abroad. He is TPC of various IEEE sponsored conferences in India and abroad. He is a reviewer/editorial board member of various international journals, e.g. IEEE Transactions on Vehicular Technology, IEEE Transactions on wireless communications, Journal of Network and Computer Applications (Elsevier), Wireless Personal Communications (Springer), Future Generation Computer Systems (Elsevier), Computers and Security (Elsevier), Mathematical and Computer Modeling (Elsevier), Information Fusion (Elsevier), EURASIP Journal of Wireless Communication and Networking (Hindawi), International Journal of Network Security (IJNS), Journal of Emerging Trends in Web Intelligence, Journal of Advances in Information Technology, IJCA and many more. He has Edited/Editing special issue of six international journals from Springer, Inderscience, and Academy publishers. He is a senior member of ACEEE and IACSIT. 


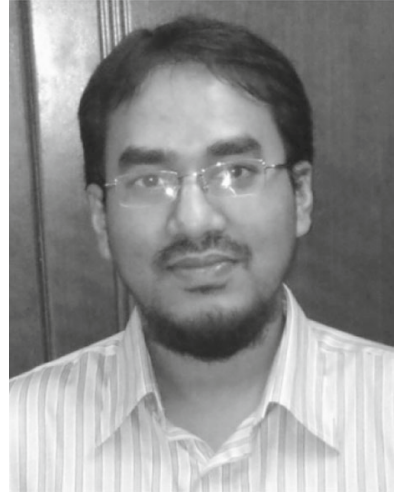

Al-Sakib Khan Pathan received Ph.D. degree in Computer Engineering in 2009 from Kyung Hee University, South Korea. He received B.Sc. degree in Computer Science and Information Technology from Islamic University of Technology (IUT), Bangladesh in 2003. He is currently an Assistant Professor at Computer Science department in International Islamic University Malaysia (IIUM), Malaysia. Till June 2010, he served as an Assistant Professor at Computer Science and Engineering department in BRAC University, Bangladesh. Prior to holding this position, he worked as a Researcher at Networking Lab, Kyung Hee University, South Korea till August 2009. His research interest includes wireless sensor networks, network security, and e-services technologies. He is a recipient of several awards/best paper awards and has several publications in these areas. He has served as a Chair, Organizing Committee Member, and Technical Program Committee member in numerous international conferences/workshops like GreenCom, HPCS, ICA3PP, IWCMC, VTC, HPCC, IDCS, etc. $\mathrm{He}$ is currently serving as an Area Editor of IJCNIS, Editor of IJCSE, Inderscience, Associate Editor of IASTED/ACTA Press IJCA and CCS, Guest Editor of some special issues of top-ranked journals, and Editor/Author of 10 books. He also serves as a referee of some renowned journals. He is a member of Institute of Electrical and Electronics Engineers (IEEE), USA; IEEE Communications Society, USA; IEEE ComSoc Bangladesh Chapter, and several other international professional organizations.

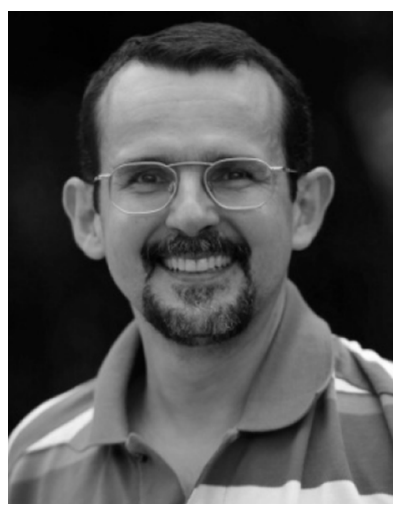

Elias P. Duarte Jr. is Associate Professor at Federal University of Parana (UFPR), Curitiba, Brazil, and chair of the Brazilian National Laboratory on Computer Networks (LARC). He obtained his Ph.D. in Computer Science from Tokyo Institute of Technology, Japan, 1997, M.Sc. in Telecommunications from the Polytechnical University of Madrid, Spain, 1991, and B.Sc. and M.Sc. in Computer Science from Federal University of Minas Gerais, Brazil, 1987 and 1991, respectively. Research interests include Computer Networks and Distributed Systems, their Dependability Management, and Algorithms. Prof. Elias has published more than 100 peer-reviewed papers, and has supervised more than 100 students, including graduate and undergraduate levels. He has served on several Editorial Boards, and chaired and served on the TPC of several conferences and workshops in his fields of interest. He chaired the Special Interest Group on Fault Tolerant Computing of the Brazilian Computing Society (2005-2007); the Graduate Program in Computer Science of UFPR (2006-2008). He is a member of the Brazilian Computer Society and the IEEE.

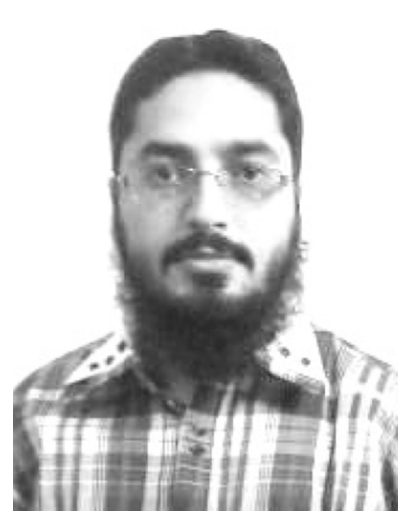

Riaz Ahmed Shaikh is Assistant Professor at Computer Science Department in King Abdulaziz University, Jeddah, Saudi Arabia. He obtained his $\mathrm{Ph} . \mathrm{D}$. in Computer Science from Kyung Hee University, Korea, 20009, M.S. in Information Technology from the National University of Sciences and Technology (NUST), Pakistan, 2005, and B.Sc. in Computer Engineering from Sir Syed University of Engineering \& Technology, Pakistan, Feb. 2003. His research interest includes privacy, security, trust management, wireless sensor networks, and vehicular networks. He is a reviewer/ editorial board member of various international journals, e.g., IEEE Transaction on Parallel and Distributed Systems, IEEE Computer Journal, Elsevier International Journal of Systems, Control and Communications, Elsevier Mathematical and Computer Modeling, Journal of Communications and Networks, Oxford Computer Journal, Transactions on Emerging Telecommunications Technologies, International Journal of Internet and Distributed Systems, and many more. He is a member of the ICST and the ACM. For more information please visit http://sites.google. com/site/riaz289/ . 